\title{
Video Prototyping of Dog-Inspired Non-verbal Affective Communication for an Appearance Constrained Robot
}

\author{
Dag Sverre Syrdal, Kheng Lee Koay, Márta Gácsi, Michael L. Walters and Kerstin Dautenhahn
}

\begin{abstract}
This paper presents results from a video humanrobot interaction (VHRI) study in which participants viewed a video in which an appearance-constrained Pioneer robot used dog-inspired affective cues to communicate affinity and relationship with its owner and a guest using proxemics, body movement and orientation and camera orientation. The findings suggest that even with the limited modalities for nonverbal expression offered by a Pioneer robot, which does not have a dog-like appearance, these cues were effective for nonverbal affective communication.
\end{abstract}

\section{INTRODUCTION}

$\mathrm{T}$ he work presented in this paper has been conducted as part of the EU FP7 Integrated project LIREC (LIving with Robots and intEractive Companions) [1]. The aims of this project are to investigate the theoretical aspects of artificial companions and embody these aspects into robust and innovative technologies, both in the form of virtual agents as well as physical robots. This also allows for studying the applicability of these theories in actual social environments which include these artifacts and is aimed at facilitating the creation of artificial companions which are suitable for long-term interactions. One of the ways in which this projects aims to fulfill its goals is to allow the artificial companions the capability of migration. This is when a single agent can transfer from one embodiment to another, including embodied conversational agents on computers and smartphones and a range of physically embodied robot platforms. This migration capability will, in addition to enhancing agent portability, also improve the range of tasks that such an agent can perform for its users, while reducing the load on the users in terms of training or customizing their preferences for each platform that the agent can inhabit.

This presents considerable technological challenges; in particular with regard to how a single high-level agent 'mind' can effectively interact with a diverse set of platforms. However, there are also concerns regarding how

Manuscript received March 1, 2010. This research was conducted as part of the EU Integrated Project LIREC (LIving with Robots and intEractive Companions) and funded by European Commision FP7-ICT under contract FP7-215554.

D.S. Syrdal, K. Dautenhahn, K.L.Koay, M.L.Walters are with the Adaptive Systems Research Group, School of Computer Science, University of Hertfordshire, Hatfield, Herts, UK; \{d.s.syrdal: k.dautenhahn; k.1.koay; m.1.walters\}@herts.ac.uk

M. Gácsi is with the Ethology Department at Eötvös Loránd University, Budapest, Hungary; gm.art@t-online.hu agent migration across different embodiments will impact the agent's interactions with users, and thus the emergent relationship between the users and the companion. A key challenge for the LIREC project will be to find ways for the agent to present a cohesive identity to its users across embodiments so that the user will find it credible that they are interacting with the same agent across embodiments. This is particularly important if the agent has a long shared interaction history with, and is therefore to be trusted, by the user.

Previous work on maintaining the identity of an agent across different embodiments tends to have focused on cues in the agent's appearance. For instance, Martin et al [2, 3 ] investigated the efficacy of different visual cues to an agent's identity across embodiments, while others such as Yasayuki and Kenshin [4] allowed the agent a screendisplay in all its embodiments which showed the name the owner had associated with a given agent.

However, the LIREC project uses both screen based agents and a range of different robots with a wide range of constraints. As such it is difficult to find one easily recognizable cue that would be uniform across these embodiments. In the University of Hertfordshire Robot House demonstration showcase, an outcome planned for the end of the LIREC project (using "robot companion in the home" scenarios), it is already planned to use commercially available research robots including PeopleBots ${ }^{1}$, Pioneers as well as Sony AIBO robots.

Lacking an easily available dimension of visual cues that can be varied across embodiments, we propose that one salient way for the user to recognise 'their' agent in a given embodiment is for it to adopt behavioral strategies to convey a sense of relationship. This could be either by referring to past interaction histories explicitly (analogous to what is being described in [5]), or implicitly through non-verbal cues.

The use of non-verbal cues to convey a sense of shared history and relationship in order to build and maintain relationships between an agent and its user has been addressed by Bickmore et al. [6-9] which has proposed the use of such strategies and demonstrated the impact of their

1 The Pioneer and Peoplebot are robot platforms commercially available from MobileRobots ( http://www.activrobots.com/) 
use with anthropomorphic virtual conversational agents. Others have also highlighted the appropriate use of nonverbal cues as important for human-robot interactions, for example, $[10,11]$. However, as Bickmore ([7]) points out, the use of human relational cues, to a large extent, depend on the need for an embodiment that has similar gestural capabilities as a human body. Following this, robots intended to communicate affective information tend to have a humanoid shape., as exemplified by the Tapus and Mataric' [12] Hands-off Therapy Robot, which was proposed as a testbed for investigating the expression of empathy, Robots like Kismet [13] also rely heavily on exaggerated anthropomorphic features for affective communication with its interactants.

However, there are, potential problems with the use of anthropomorphic cues. Dautenhahn [14] argues that humanoid robots will be likely to raise the expectations of the user in terms of its capabilities and interactional

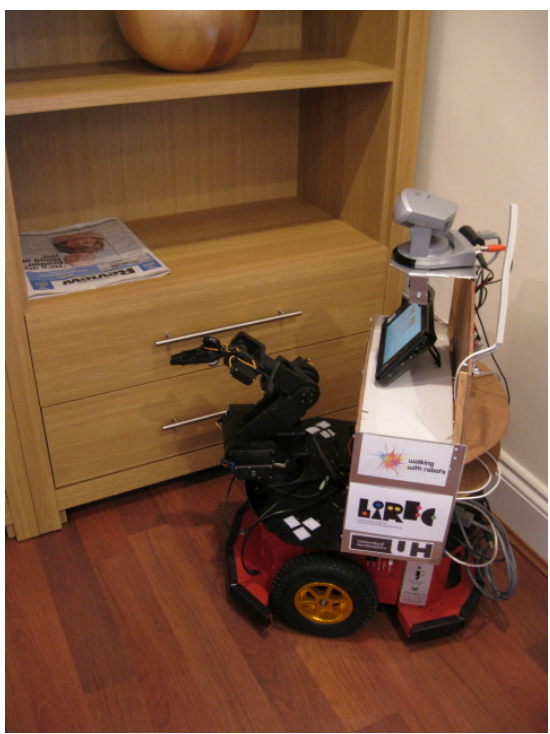

Fig 1 Pioneer Robot used in the Video

affordances, and that the robot's (likely) inability to fulfill these expectations will be damaging to interactions.

Thus, it is no surprise, that the most iconic interactional robots intended for mass use by the public have not, in fact, relied on anthropomorphic cues, but on cues inspired by animals. The entertainment robots AIBO and Pleo, both use zoomorphic, (animal-like) attributes, to engage with their users. Despite the lack of humanoid affordances, users of both robots report that their behaviour can be highly evocative in terms of the emotions they convey $[15,16]$. Partly, their success (in terms of interaction, if not in sales) can be attributed to the way that the zoomorphic affective cues are effective, while at the same time managing the user's expectations as to its capabilities. In assistive robotics, the wide-spread adoption of the seal like robot Paro [17] can be understood as the result of it allowing an emotionally rich interaction, without raising high expectations as to the capabilities of the robot.

This suggests that there is a wide range of emotive behaviours which can be utilised to maintain and reinforce the emergent relationship between the agent and its user but is not reliant on human-specific expressive capabilities,.

However, robots designed for purposes other than being purely interactional artefacts, often have their appearance constrained by their function, and so have fewer avenues of non-verbal communication. This issue was highlighted by Bethel \& Murphy [18], in their survey of affective communication for appearance-constrained robots. This survey suggests that the use of body orientation, camera movement and proxemics can be used effectively for nonverbal communication. Another study [19] by the same group suggests that emotive behaviours are effective in eliciting an appropriate response from the user, despite the lack of an appearance familiar to the user.

Based on the above findings, we propose that is possible to use behaviours available to a mechanical-looking Pioneer embodiment, such as proxemic behaviour, gross body movements and camera orientation and movement, in order to convey a sense of shared interaction history to the user. Drawing on the earlier noted success of zoomorphic robots, the use of animal-inspiration behaviours is a valid avenue of investigation. We decided to base our interactional behaviours on what is observed in dog-human interactions.

Dogs and humans share a long history, genetic evidence suggests that the domestic dog has accompanied humans for possibly 15-20 000 years [20], engaged in variety of tasks in different contexts and cultures. The selectional processes during this joint history of the two species have lead to dog which has unique capabilities in both understanding human socio-communicative signals and displaying affective behaviours which humans comprehend naturally $[21,22]$. Also, the continued popularity of dog ownership in most cultures means that potential users of an artificial agent are likely to have had some exposure to dogs and their means of communication.

In HRI, specifically using a medium size dog scaled Pioneer robot, dog-like behaviour has previously been considered by Nicolescu \& Matarić [24] as a means for a robot to direct human attention and movement. While these cues were not specifically intended for emotive purposes, they still suggest that such cues can be effective. 


\section{II.METHODOLOGY}

\section{A. Initial Development of the Videos}

Due to the exploratory nature of the study, a video human-robot interaction study was decided upon. As we have noted earlier [24-26] using video rather than live interactions for evaluating prototype behaviours allows for a focused investigation into specific issues involved in the development of a system, without requiring a fully functional platform.

The study videos were developed in co-operation with members of the University of Hertfordshire School of Computer Science in UK, and the Department of Ethology at Eötvös Loránd University in Hungary. This ensured both a technically realistic repertoire of robot behaviours as well as allowing the robot to use ethologically sound behaviours, analogous to those displayed by dogs for affective communication. Note, the pioneer robots (see figure 1) are not dog-like in appearance and lacks both ears and a tail which are important in dog affective expressiveness.

A second set of two videos were also produced during this process which showed dogs trained to help people in wheelchairs. These videos served two functions, they were used in finalising the robot's behaviours as well as allowing for comparisons between dogs and robots in future evaluation of the behaviours. In order to create a rich and realistic context for the behaviours, the fictional narrative of the video centered around an owner of the robot returning home and receiving a guest, with the robot assisting with the task of transporting food and beverages from the kitchen to the dining room. This scenario allowed for several of interactions with both humans.

The rough storyline of the videos is detailed below in Table 1:

Table 1 Video Storyline

\begin{tabular}{|c|c|}
\hline Scene No. & Brief Description \\
\hline 1 & $\begin{array}{l}\text { Robot/Dog is in dining room, Owner enters from } \\
\text { outside, robot greets owner. }\end{array}$ \\
\hline 2 & $\begin{array}{l}\text { Robot/Dog is in dining room, Guest enters from } \\
\text { outside, robot/dog greets guest and uses social } \\
\text { referencing to interact with owner. }\end{array}$ \\
\hline 3 & $\begin{array}{l}\text { Robot/Dog follows owner to the kitchen and is } \\
\text { loaded with items for tea and biscuits. }\end{array}$ \\
\hline 4 & $\begin{array}{l}\text { Robot/Dog attempts to gain guests attention for } \\
\text { help in unloading. }\end{array}$ \\
\hline 5 & $\begin{array}{l}\text { Owner and Guest have tea and converse with robot } \\
\text { /dog watching. }\end{array}$ \\
\hline 6 & $\begin{array}{l}\text { Guest leaves, robot/dog engages in "farewell" } \\
\text { behaviour with guest. }\end{array}$ \\
\hline
\end{tabular}

In this video, scenes 1,2 , and 6 were the most important in terms of examining the efficacy and legibility of the robot's non-verbal cues in order to differentiate between the guest and owner in terms of interaction history and relationship. Scenes 3,4 and 5 were intended to situate the interactions within the everyday experience of the characters in the video.

\section{B. Robot Behaviours}

We will here consider the non-verbal affective behaviours the robot used to differentiate between guest and owner. Greeting and "farewell" were chosen due to their importance in framing interactions within a larger timeframe [9].

The greeting behaviour for the owner consisted of the robot moving rapidly towards the owner as she entered, orienting its camera briefly towards the face of the owner and then moving away in the direction the owner would later move towards. This behaviour was intended to communicate enthusiasm both in terms of greeting the owner and aiding in the tasks the owner was later to perform.
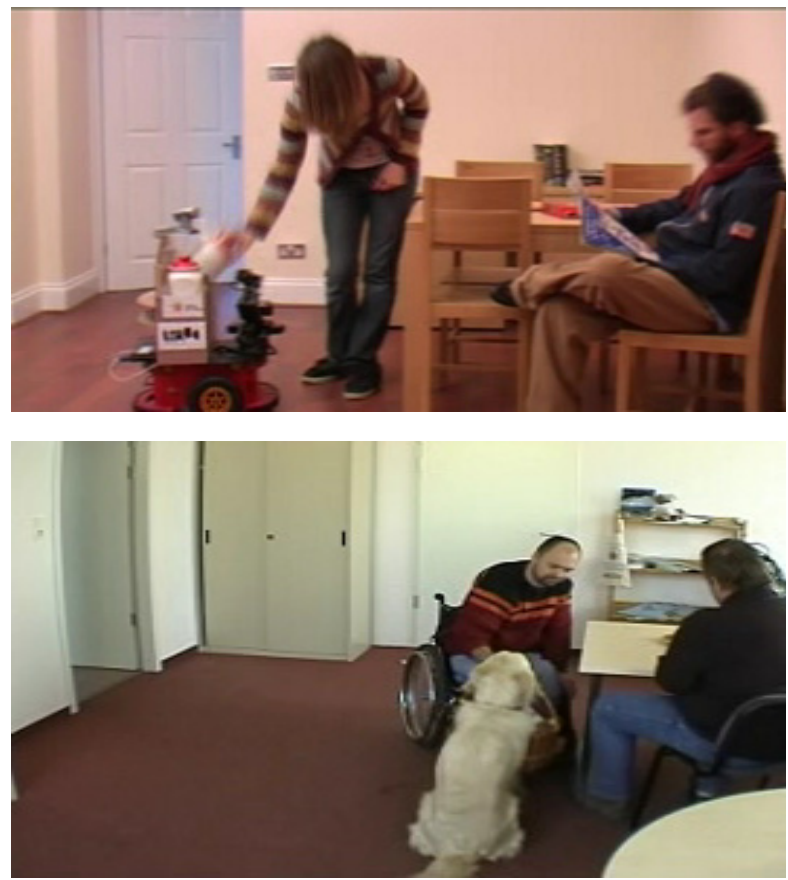

Fig 2 Fetch and Carry task. Robot (Top) and Dog(Bottom) being offloaded in the dining room.

The greeting behaviour for the guest similarly included the robot moving towards the guest, but the robot's camera spent more time observing the user. Also, this greeting behaviour also included social referencing by the robot orienting itself and its camera in the direction of the owner 


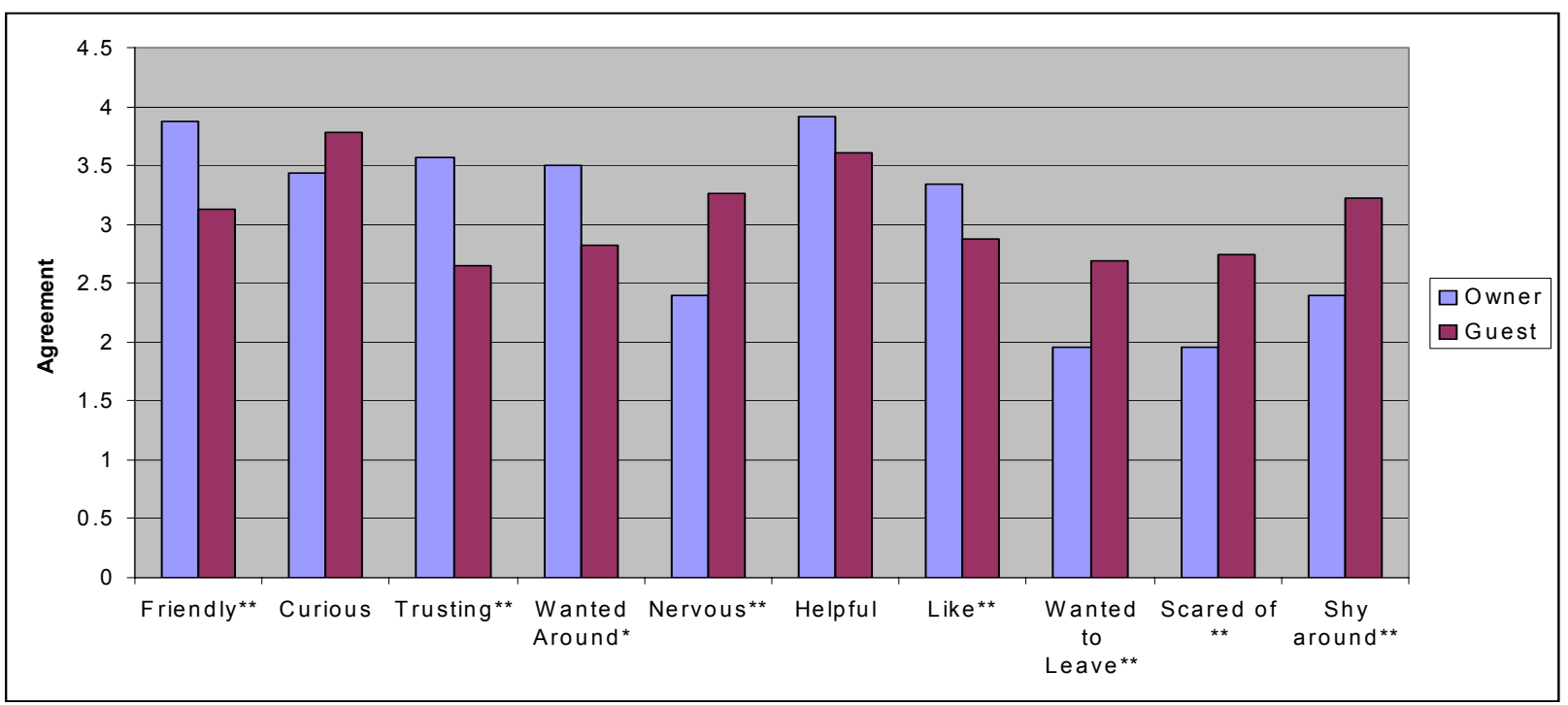

Fig 3. Mean responses to statement regarding the robot regarding the owner and dog on a 5-point likert scale in which 1 indicates complete disagreement and 5 complete agreement. *, Significance of Mean Difference $\mathrm{p}<.05$.; **, Signficance of Mean Difference $\mathrm{p}<.01$.

immediately. This behaviour was intended to mirror the way that dogs examine new humans in their home environment and also the way that dogs take cues from humans with which they have an established relationship to decide on appropriate behaviour to novel events and people in their environment.

The "farewell" behaviour towards the guest consisted of the robot orienting its body and camera towards the guest, observing him as he left the room. The "farewell" behaviour towards the owner consisted of the robot orienting its body towards the owner and then moving towards the owner as she walked to the door, following the owner to the door, only stopping as it reached the door.

It is important to note that "farewell" behaviour in dogs may, strictly speaking, not be communicating a farewell in the human sense, but is merely the dog communicating a desire for being constantly near to the owner. Its role in the human-robot interaction is intended to fulfill the function it would have in human-human interactions.

\section{Qualitative Pilot Study}

A small-scale pilot study using 5 participants was conducted. This, similarly to previous work on video prototyping [24], was done to qualitatively assess the efficacy of the robot behaviours, as well as gaining insight into how participants might describe the behaviours. In this study, the participants watched the videos, followed by an explicitation interview [27] focusing on their experience of watching the video. Results from this study were encouraging. Participants, without prompting, referred to the robot's behaviour as signaling affinity and relationship. In some cases, they also related it to their own experience with dogs. After this interview, the participants were also shown a video with a dog performing the same tasks.

\section{Quantitative Study}

Following the qualitative pilot study, a questionnaire was designed based on descriptive statements from the participants in the pilot study regarding the robot's emotional response to, and relationship with the owner and the guest in the video. (See table 2 for the list of statements). There were also open-ended questions allowing them to describe their impression of the robot's behaviour towards the guest and owner. The video was shown to 23 participants (ages 15-18, 24 male, 2 female) who attended a Robotic Visions event in Oxford ${ }^{2}$. The participants watched the video of the human-robot interaction and were then invited to complete a questionnaire. Following the questionnaire, a video showing a dog performing the same tasks in the same context was shown. After the second video, the researchers led a short discussion with the participants around the differences between the way the dog and the robot behaved in the videos. This discussion was informed by the pilot study, but as the researchers were sensitive to the issue of participant concerns regarding dogs being replaced by robots, it was considered more helpful to conduct this comparison discussion as a dialogue rather than as a onesided questionnaire, in particular due to the aims of Robotic Visions.

\footnotetext{
2 Robotics vision is a public engagement project aiming to provide a platform of dialogue between young people, robotics researchers and policymakers.See

http://www.walkingwithrobots.org/events/RoboticVisions.php for more details.
} 


\section{RESULTS}

\section{A. Likert Scale responses}

The responses to the likert-scales were analysed using paired sample t-tests on the difference between responses regarding the robot's behaviour to the Owner and the

Table 2: Statements regarding the robot and the observed mean differences in agreement between owner and guest statements.

\begin{tabular}{|c|c|c|c|}
\hline $\begin{array}{l}\text { Statement: The } \\
\text { Robot seemed } \\
\text { to... }\end{array}$ & $\begin{array}{l}\text { Mean } \\
\text { Difference } \\
\text { (SE) }\end{array}$ & $\begin{array}{l}\text { T-value } \\
(\mathrm{Df}=22)\end{array}$ & Significance \\
\hline $\begin{array}{l}\text {...be friendly } \\
\text { towards X }\end{array}$ & $.74(.16)$ & 4.75 & $.000 * *$ \\
\hline $\begin{array}{l}\ldots \text { be curious } \\
\text { towards } X\end{array}$ & $-.34(.23)$ & -1.50 & .148 \\
\hline$\ldots$ trust $X$ & $.91(.21)$ & 4.21 & $.000 * *$ \\
\hline $\begin{array}{l}\ldots \text { want } X \\
\text { around }\end{array}$ & $.68(.25)$ & 2.73 & $.012 *$ \\
\hline $\begin{array}{l}\ldots \text { be nervous } \\
\text { around } X\end{array}$ & $-.87(.25)$ & -3.43 & $.002 * *$ \\
\hline $\begin{array}{l}\text {.. be helpful } \\
\text { towards X }\end{array}$ & $.30(.19)$ & 1.57 & .129 \\
\hline$\ldots$ like $X$ & $.48(.16)$ & 2.90 & $.008 * *$ \\
\hline $\begin{array}{l}\ldots \text { want } X \text { to } \\
\text { leave }\end{array}$ & $-.74(.17)$ & -4.38 & $.000 * *$ \\
\hline $\begin{array}{l}\ldots \text { be scared of } \\
X\end{array}$ & $-.78(.18)$ & -4.16 & $.000 * *$ \\
\hline ... shy around $X$ & $-.83(.24)$ & -3.43 & $.002 * *$ \\
\hline
\end{tabular}

Guest. These responses are shown in Table 2 and Figure 3. The responses indicate that participants found that the robot was more friendly and trusting towards the owner, as well as wanting the owner's presence to a larger degree than the guest's. Likewise, they suggest that participants also saw the robot as more nervous, scared and shy around the guest, as well as wanting the guest to leave to a larger degree than the owner. The participants did not significantly distinguish between the robot's behaviour towards owner and guest in terms of curiosity and helpfulness (The effect sizes observed for these two statements were $d=.31$ and .32 , with an observed statistical power of .41 and .45, respectively).

\section{B. Open-ended Responses and Subsequent Discussion}

The open-ended responses of the participants also distinguished clearly between the guest and the owner in terms of the robot's behaviour. In terms of its behaviour towards the owner, 18 percent of statement referenced pets or dogs; 50 percent of statements referenced positive emotions like affection or loyalty; the remaining participants referenced task-related capabilities.

For the guest, 8 percent referenced pets; 58 percent referred to caution or uncertainty; the remaining statements referenced task-related behaviour.

The subsequent discussion focused primarily on the differences between the robot and the dog. The primary interest of the group revolved around agency and authenticity. This suggested that while the robot's behaviour was legible in terms of its communicative power, the emotional impact of this communication was less than that performed by a dog.

\section{DISCUSSION AND CONCLUSIONS}

The findings presented in this paper support the further investigation of the use of cues inspired by dog interactions with humans for the establishment and maintenance of human-robot relationships even for appearance constrained robots. It suggests that these cues do not rely solely on a dog-like shape, but may be legible to naïve interactants even when performed by an appearance-constrained robot with a mechanoid appearance [28] if presented within the appropriate context of use. This suggests that such robots may use similar relationship building strategies as suggested by Bickmore et al. [6] for humanoid embodied conversational agents. However, we can make no claims as to the efficacy of the cues utilized by the robot outside of this study. If the robot's behaviours were presented outside of a given context such as the one in the video, they might not give viewers the same impressions. We would like to point out, however, that cues such as these would only be useful for us within the context of a situated human-robot interaction, and that for this, these cues will be able to maintain the notion of an existing interaction history between user and robot, thus allowing an agent to maintain its identity across embodiments using these cues for robotic embodiments such as the pioneer.

As such, these findings will be useful for informing future work on long-term relationships between robots and their human users. An open question for future work is to what extent these cues enhance interaction between the robot and the user. The issues of agency and authenticity, also noted by amongst others, Turkle [29] was readily available in the reasoning of the participants. As such the impact of such cues, beyond legibility, in terms of relationship maintenance can only be assessed through long-term, live human-robot interaction studies over a prolonged period of time. The findings presented here provide solid justification for the use of dog-inspired cues in such studies.

\section{V.ACKNOWLEDGEMENTS}

The authors would like to thank the Ethology Department at Eötvös Loránd University, in particular Adam Miklosi, Enikő Kubinyi and Gabriella Lakatos for their aid in creating the videos used for this study as well as for their continued help in conducting the study. 


\section{REFERENCES}

[1] LIREC, http://www.lirec.org, 2008.

[2] A. Martin, G. M.P.O'Hare, B. R. Duffy, B. Schön, and J. F. Bradley, "Maintaining the Identity of Dynamically Embodied Agents," The Fifth International Working Conference on Intelligent Virtual Agents - IVA 2005 Kos, Greece, September 2005, 2005.

[3] A. Martin, "Martin, A. (2007). Dynamically Embodied Virtual Agents," PhD Thseis, School of Computer Science and Informatics. University College Dublin: Dublin, 2007.

[4] S. Yasuyuki and M. Kenji, "AgentSalon: facilitating face-to-face knowledge exchange through conversations among personal agents," Proceedings of the fifth international conference on Autonomous agents, ACM, 2001., 2001.

[5] R. Anderson, "Defining the Supernatural in Iceland," Anthropological Forum, vol. 13, pp. 125-130, 2003.

[6] T. W. Bickmore and R. W. Picard, "Establishing and Maintaining Long-Term Human Computer Relationships," ACM Transactions on Computer-Human Interaction, vol. 12, pp. 293-327, 2005.

[7] T. W. Bickmore and J. Cassell, "Social Dialogue with Embodied Conversational Agents," in Natural, Intelligent and Effective Interaction with Multimodal Dialogue Systems, J. v. Kuppevelt, L. Dybkjaer, and N. Bernsen, Eds. New York: Kluwer Academic, 2005, pp. 23-54.

[8] T. W. Bickmore and J. Cassell, "Relational Agents: A Model and Implementation of Building User Trust," Proceedings of the SIGCHI conference on Human factors in computing systems, pp. 396-403, 2001.

[9] T. W. Bickmore, L. Caruso, K. Clough-Gorr, and T. Heeren, "'It's just like you talk to a friend' relational agents for older adults," Interacting With Computers, vol. 17, pp. 711-735, 2005.

[10] A. Tapus and M. J. Mataric', "Towards Socially Assistive Robotics," International Journal of the Robotics Society of Japan, vol. 24, 2006.

[11] K. Dautenhahn, "Robots We Like to Live With? - A Developmental Perspective on a Personalized, Life-Long Robot Companion," Proceedings of the 13th IEEE International Workshop on Robot and Human Interactive Communication(RO-MAN 2004), pp. 17-22., 2004.

[12] A. Tapus and M.J. Mataric, "Emulating empathy in socially assistive robotics.," Proceedings of the American Association of Artificial Intelligence (AAAI) Spring Symposium on - Multidisciplinary Collaboration for Socially Assistive Robotics, Palo Alto,USA, 2007., 2007.

[13] C. L. Breazal, Designing Sociable Robots. Cambridge, Massachusetts: MIT Press, 2002.

[14] K. Dautenhahn, "Design spaces and niche spaces of believable social robots," Proceedings of the 11th IEEE International Workshop on Robot and Human Interactive Interactive Communication(RO-MAN 2002), Berlin, Germany Sept 25-27, 2002, 2002.

[15] M. Jacobsson, "Play, Belief and Stories about Robots: A Case Study of a Pleo Blogging Community," Proceedings of the IEEE International Symposium on Robot and Human Interactive Communication (Ro-Man 2009), 2009.

[16] B. Friedman, P. H. Kahn, and J. Hagman, "Hardware companions?What online AIBO discussion forums reveal about the humanrobotic relationship," Proceedings of the CHI 2003 Conference on Human Factors in Computing Systems. New York: ACM, 2003.

[17] T. Shibata, K. Wada, T. Saito, and K. Tanie, " Human interactive robot for psychological enrichment and therapy.," Proceedings of the AISB '05 Symposium on Robot Companions: Hard Problems and Open Challenges in Robot-Human Interaction Hertfordshire, England, April 12-15, 2005). Hertfordshire, England: University of Hertfordshire, 2005, 98-109., 2005.

[18] C. L. Bethel and R. R. Murphy, "Survey of Non-facial/Non-verbal Affective Expressions for Appearance-Constrained Robots.," IEEE Transactions on Systems, Man, and Cybernetics - Part C: Applications and Reviews, vol. 38, 2008.
[19] C. L. Bethel, K. Salomon, and R. R. Murphy, "Preliminary Results: Humans Find Emotive Non-Anthropomorphic Robots More Calming," 4th ACM/IEEE International Conference on HumanRobot Interaction 2009, San Diego, CA, March, 2009.

[20] Ostrander, E.A. Giger, U., and Lindbladh, K., eds. The dog and its genome, pp. 119-140. Cold Spring Harbor Laboratory Press, New York.

[21] Topál, J., Miklósi, Á., Gácsi, M., Dóka, A., Pongrácz, P., Kubinyi, E., Virányi Zs., Csányi, V. 2009. The dog as a model for understanding human social behavior. Advances in the Study of Animal Behaviour, 39: 71-116.

[22] P. Pongrácz, C. Molnár, and Á. Miklósi, "Acoustic parameters of dog barks carry emotional information for humans," Applied Animal Behaviour Science, vol. 100, pp. 228, 2006. [

[23] M.N. Nicolescu, and M. J. Mataric, "Linking Perception and Action in a Control Architecture for Human-Robot Domains," Proceedings Thirty-Sixth Hawaii International Conference on System Sciences, HICSS-36, Hawaii, USA, January 6-9, 2003. 2003.

[24] D. S. Syrdal, N. R. Otero, and K. Dautenhahn, "Video Prototyping in Human-Robot Interaction: Results from a Qualitative Study," Proceedings of the 2008 European Conference on Cognitive Ergonomics, 2008.

[25] S. Woods, M. Walters, K. L. Koay, and K. Dautenhahn, "Comparing Human Robot Interaction Scenarios Using Live and Video Based Methods: Towards a Novel Methodological Approach," Proc. $A M C^{\prime} 06$, The 9th International Workshop on Advanced Motion Control, Istanbul March 27-29, pp. 750-755, 2006.

[26] S. N. Woods, M. L. Walters, K. L. Koay, and K. Dautenhahn, "Methodological Issues in HRI: A Comparison of Live and VideoBased Methods in Robot to Human Approach Direction Trials," Proceedings, 15th IEEE International Workshop on Robot and Human Interactive Communication (RO-MAN2006), pp. 51-58, 2006.

[27] A. Light, "Adding method to meaning: A Technique for exploring peoples' experiences with technology," Behaviour \& Information Technology, vol. 25, pp. 175-187, 2006

[28] M. Walters, D. S. Syrdal, K. Dautenhahn, R. T. Boekhorst, K. L. Koay, and S. Woods, "Avoiding the Uncanny Valley - Robot Appearance, Personality and Consistency of Behavior in an Attention-Seeking Home Scenario for a Robot Companion," Autonomous Robots, vol. 24, pp. 159-178, 2008.

[29] S. Turkle, W. Taggart, C. D. Kidd, and O. Dasté, "Relational artifacts with children and elders: the complexities of cybercompanionship," Connection Science, vol. 18, pp. 347-361, 2006 